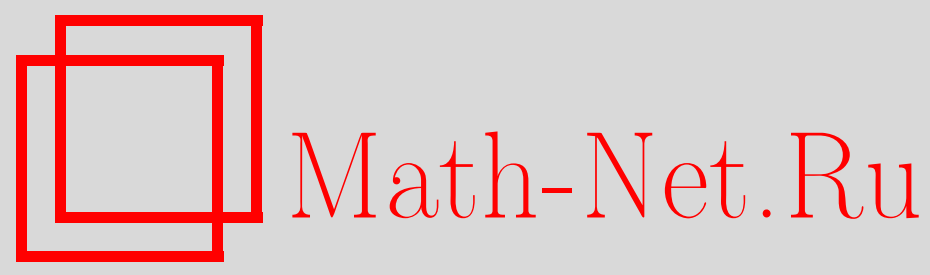

В. А. Добрынский, О структуре фазового портрета одного эндоморфизма плоскости в момент бифуркации его диагонального аттрактора, Матем. заметки, 2003, том 74, выпуск $2,230-237$

DOI: https://doi.org/10.4213/mzm255

Использование Общероссийского математического портала Math-Net.Ru подразумевает, что вы прочитали и согласны с пользовательским соглашением http://www . mathnet.ru/rus/agreement

Параметры загрузки:

IP : 3.89 .197 .203

26 апреля 2023 г., $16: 26: 46$

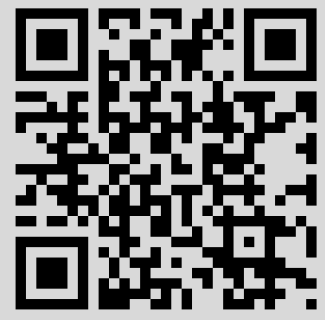




\section{О СТРУКТУРЕ ФАЗОВОГО ПОРТРЕТА ОДНОГО ЭНДОМОРФИЗМА ПЛОСКОСТИ В МОМЕНТ БИФУРКАЦИИ ЕГО ДИАГОНАЛЬНОГО АТТРАКТОРА}

\section{В. А. Добрынский}

Изучены явления, происходящие в момент существенно двумерной бифуркации неподвижной точки, вложенной в диагональный аттрактор двумерного отображения, построенного сцеплением пары идентичных одномерных унимодальных кусочно-линейных отображений.

Библиограбоия: 12 названий.

Одним из наиболее изученных классов одномерных систем является семейство кусочно-линейных отображений вида $\tau \rightarrow 1-a|\tau|$. Это связано с тем, что, обладая по сути дела столь же сложной динамикой, как и гладкие отображения, кусочно-линейные намного проще и легче поддаются изучению аналитическими методами.

В последнее время в литературе широко обсуждаются эффекты, возникающие при сцеплении ряда идентичных одномерных отображений.

Пусть $f_{a}: \tau \rightarrow f_{a}(\tau)$ - однопараметрическое семейство отображений прямой $\mathbb{R}^{1}$ в себя. Сцепление пары таких отображений может быть произведено различными способами. Чаще всего рассматриваются отображения вида

$$
(x, y) \rightarrow\left(f_{a}(x)+\zeta\left(f_{a}(y)-f_{a}(x)\right), f_{a}(y)+\zeta\left(f_{a}(x)-f_{a}(y)\right)\right),
$$

где $(x, y) \in \mathbb{R}^{2}$. Очевидно, они обладают свойством симметрии (относительно замен $x \rightarrow y, y \rightarrow x)$, вследствие чего они при любых значениях параметров $a$ и $\zeta$ имеют одномерное инвариантное многообразие в виде главной диагонали $x=y$, на которой они совпадают с $f_{a}$. Ниже исследуются отображения (1) с $f_{a}(\tau)=1-a|\tau|$, т.е. рассматривается двупараметрическое семейство кусочно-линейных эндоморфизмов плоскости вида

$$
\Phi:(x, y) \rightarrow(1-a(1-\zeta)|x|-a \zeta|y|, 1-a(1-\zeta)|y|-a \zeta|x|),
$$

где $a$ и $\zeta$ - параметры такие, что $0<a<2$ и $0<\zeta<1$.

Очевидно, $T=(1 /(1+a), 1 /(1+a))$-неподвижная точка эндоморфизма $\Phi$. Через нее проходит прямая $y=2 /(1+a)-x$, отрезок $\{(x, y): 0 \leqslant x \leqslant 2 /(1+a), y=2 /(1+a)-x\}$ которой является инвариантньм множеством для $\Phi$. В самом деле, так как $x \geqslant 0$ и $2 /(1+a)-x \geqslant 0$ при $0 \leqslant x \leqslant 2 /(1+a)$, то

$$
\Phi:\left(\begin{array}{c}
x \\
\frac{2}{1+a}-x
\end{array}\right) \rightarrow\left(\begin{array}{c}
1-a(1-2 \zeta) x-2 \zeta \frac{a}{1+a} \\
1+a(1-2 \zeta) x-2(1-\zeta) \frac{a}{1+a}
\end{array}\right)=\left(\begin{array}{c}
\widetilde{x} \\
\widetilde{y}
\end{array}\right) .
$$


Откуда $\widetilde{y}=2 /(1+a)-\widetilde{x}$.

При $a(1-2 \zeta)=1$ выполнено $\widetilde{y}=x$, а $\widetilde{x}=y$. В этом случае данньй отрезок целиком состоит из периодических периода 2 точек эндоморфизма $\Phi$. Обозначим через

$$
T_{ \pm}^{(\alpha)}=\left(\frac{1}{1+a} \mp \frac{\alpha}{2}, \frac{1}{1+a} \pm \frac{\alpha}{2}\right)
$$

периодические периода 2 точки, принадлежашие прямьм $y=x \pm \alpha$.

Численньми методами свойства отображения (1) и ему подобных исследовались во многих работах (см., например, [1]-[3]), но аналитических результатов известно мало. В частности, в [4] и независимо в [5] доказано, что для любого $(1+\sqrt{5}) / 2<a<2$ существует $\zeta_{a}>0$ такое, что при любом $\zeta \in\left(0, \zeta_{a}\right)$ отображение $\Phi$ имеет хаотический аттрактор $\Lambda$, на котором $\Phi$ является топологически перемешиваюшим отображением. Кроме того, в [5] установлено, что этот аттрактор $\Lambda$ представляет собой четырехугольник (ромбоид), стороны которого образованы парами отрезков двух первых образов критического множества отображения $\Phi$. (Напомним, что критическим для отображения $\Psi$ называется множество $K_{\Psi}$ точек, в которых якобиан $D \Psi$ обрашается в нуль или же вообще не существует [6], [7]). С другой стороны, почти очевидно, что при $\zeta<1 / 2$ достаточно близких к $1 / 2$ упомянутый выше аттрактор $\Lambda$ принадлежит диагонали $x=y$, совпадая с отрезком $\left[\Phi^{2}(O), \Phi(O)\right]$, где $O=(0,0)$ - начало системы координат.

Teоpema 1. Ecлu $a \in(1,2)$ u $\zeta \in((a-1) /(2 a), 1 / 2)$, mo $\Lambda \stackrel{\text { def }}{=}\left[\Phi^{2}(O), \Phi(O)\right]-$ асимптотически устойчивое множество.

ДокАЗАТЕльство. В самом деле, пусть $\alpha>0$. Вычисляя, нетрудно убедиться в наличии следуюших соотношений:

$$
\begin{aligned}
& \Phi(\{(x, y): y=x+\alpha, x \geqslant 0\})=\{(x, y): y=x-a(1-2 \zeta) \alpha, x \leqslant 1-a \zeta \alpha\}, \\
& \Phi(\{(x, y): y=x+\alpha, x \leqslant-\alpha\})=\{(x, y): y=x+a(1-2 \zeta) \alpha, x \leqslant 1-a(1-\zeta) \alpha\}, \\
& \Phi(\{(x, y): y=x+\alpha,-\alpha<x<0\})=\{(x, y): y=2-x-a \alpha, \\
&1-a(1-\zeta) \alpha<x<1-a \zeta \alpha\}, \\
& \Phi(\{(x, y): y=x-\alpha, x \geqslant \alpha\})=\{(x, y): y=x+a(1-2 \zeta) \alpha, x \leqslant 1-a(1-\zeta) \alpha\}, \\
& \Phi(\{(x, y): y=x-\alpha, x \leqslant 0\})=\{(x, y): y=x-a(1-2 \zeta) \alpha, x \leqslant 1-a \zeta \alpha\}, \\
& \Phi(\{(x, y): y=x-\alpha, 0<x<\alpha\})=\{(x, y): y=2-x-a \alpha, \\
&1-a(1-\zeta) \alpha<x<1-a \zeta \alpha\} .
\end{aligned}
$$

Таким образом, при $\zeta \in((a-1) /(2 a), 1 / 2)$, т.е. когда $a(1-2 \zeta)<1$, эндоморфизм $\Phi$ отображает полосу $\{(x, y): x-\alpha \leqslant y \leqslant x+\alpha,-\infty<x<+\infty\}$ на часть полосы $\{(x, y): x-a(1-2 \zeta) \alpha \leqslant y \leqslant x+a(1-2 \zeta) \alpha,-\infty<x<+\infty\}$, которая, в свою очередь, располагается внутри первой полосы. И, поскольку при $j \rightarrow \infty a^{j}(1-2 \zeta)^{j} \rightarrow 0$, то

$$
\bigcap_{j=0}^{\infty} \Phi^{j}(\{(x, y): x-\alpha \leqslant y \leqslant x+\alpha,-\infty<x<+\infty\}) \subset\{(x, y): x=y,-\infty<x<+\infty\} .
$$


Как известно [2],

$$
\Phi\left(\mathbb{R}^{2}\right)=\left\{(x, y): 1+\frac{1-\zeta}{\zeta}(x-1) \leqslant y \leqslant 1+\frac{\zeta}{1-\zeta}(x-1), x \leqslant 1\right\} .
$$

Обозначим

$$
\Delta_{\alpha}=\{(x, y): x-\alpha \leqslant y \leqslant x+\alpha,-\infty<x<+\infty\} \cap B D E F,
$$

где $B D E F-$ ромб с вершинами $B=(1,1), D=(1-2 \zeta,-1+2 \zeta), E=(-1,-1)$, $F=(-1+2 \zeta, 1-2 \zeta)$, сторонами которого служат отрезки прямых

$$
\begin{aligned}
& y=1+\frac{\zeta}{1-\zeta}(x-1), \quad y=-1+\frac{\zeta}{1-\zeta}(x+1), \\
& y=1+\frac{1-\zeta}{\zeta}(x-1), \quad y=-1+\frac{1-\zeta}{\zeta}(x+1) .
\end{aligned}
$$

Так как при $1<a<2$ вьполнено $\Phi(E)=\Phi(B)=\Phi^{2}(0)=(1-a, 1-a) \in \operatorname{Int}(E O)$ и $\Phi(O)=B$, то (ввиду непрерьвности $\Phi$ ) из сказанного вытекает существование достаточных малых $\alpha$ и $\beta>0$ таких, что $\Phi\left(\operatorname{Cl}\left(U_{\beta}\left(\Delta_{\alpha}\right)\right)\right) \subset U_{\beta}\left(\Delta_{\alpha}\right)$. (Здесь и ниже $U_{\beta}(\cdot)$, $\operatorname{Int}(\cdot)$ и $\mathrm{Cl}(\cdot)$ обозначают соответственно $\beta$-окрестность, внутренность и замыкание некоторого множества.) В силу сходимости $a^{j}(1-2 \zeta)^{j} \rightarrow 0$ при $j \rightarrow \infty$ последнее означает, что

$$
\bigcap_{j=0}^{\infty} \Phi^{j}\left(\operatorname{Cl} U_{\beta}\left(\Delta_{\alpha}\right)\right)=\left[\Phi^{2}(O), \Phi(O)\right]=\Lambda .
$$

Следовательно, $\Lambda$ - асимптотически устойчивое множество (при $1<a<2$ и $(a-1) /(2 a)$ $<\zeta<1 / 2)$.

Пусть теперь $\zeta=(a-1) /(2 a)$, т.е. $a(1-2 \zeta)=1$. В этом случае $\Phi$ каждую прямую $y=x+\alpha$ отображает в ломаную $L_{\alpha}$, звеньями которой являются лучи

$$
\begin{aligned}
& L_{\alpha}^{-}=\left\{(x, y): y=x-\alpha, x \leqslant 1-(a-1) \frac{\alpha}{2}\right\}, \\
& L_{\alpha}^{+}=\left\{(x, y): y=x+\alpha, x \leqslant 1-(a+1) \frac{\alpha}{2}\right\},
\end{aligned}
$$

соединенные воедино отрезком

$$
L_{\alpha}^{\star}=\left\{(x, y): y=2-x-a \alpha, 1-(a+1) \frac{\alpha}{2}<x<1-(a-1) \frac{\alpha}{2}\right\} .
$$

Поскольку ниже рассматриваются те (и только те) участки ломаной $L_{\alpha}$, которые лежат внутри ромба $B D E F$, они обозначаются так же, как и участки ломаной, которой они принадлежат. Введем на $L_{\alpha}$ порядок следуюшим образом: если $P=\left(x_{P}, y_{P}\right) \in L_{\alpha}^{-}$, $Q=\left(x_{Q}, y_{Q}\right) \in L_{\alpha}^{\star}, R=\left(x_{R}, y_{R}\right) \in L_{\alpha}^{+}$, то $P \propto Q \propto R$; если $P, Q \in L_{\alpha}^{-}$, то $P \propto Q$ тогда и только тогда, когда $x_{P}<x_{Q}$; если $P, Q \in L_{\alpha}^{\star}$, то $P \propto Q$ тогда и только тогда, когда $y_{P}<y_{Q}$; если $P, Q \in L_{\alpha}^{+}$, то $P \propto Q$ тогда и только тогда, когда $x_{P}>x_{Q}$. Обозначим

$$
\begin{array}{lll}
A_{0}=E D \cap L_{\bar{\alpha}}^{-}, & A_{1}=\{(x, y): x=0\} \cap L_{\alpha}^{-}, & A_{2}=\{(x, y): y=0\} \cap L_{\alpha}^{-}, \\
A_{3}=B D \cap L_{\alpha}^{-}, & A_{4}=F B \cap L_{\alpha}^{+}, & A_{5}=\{(x, y): x=0\} \cap L_{\alpha}^{+}, \\
A_{6}=\{(x, y): y=0\} \cap L_{\alpha}^{+}, & A_{7}=E F \cap L_{\alpha}^{+} &
\end{array}
$$

и будем считать $A_{0}$ началом $L_{\alpha}$, а $A_{7}$ концом его. 


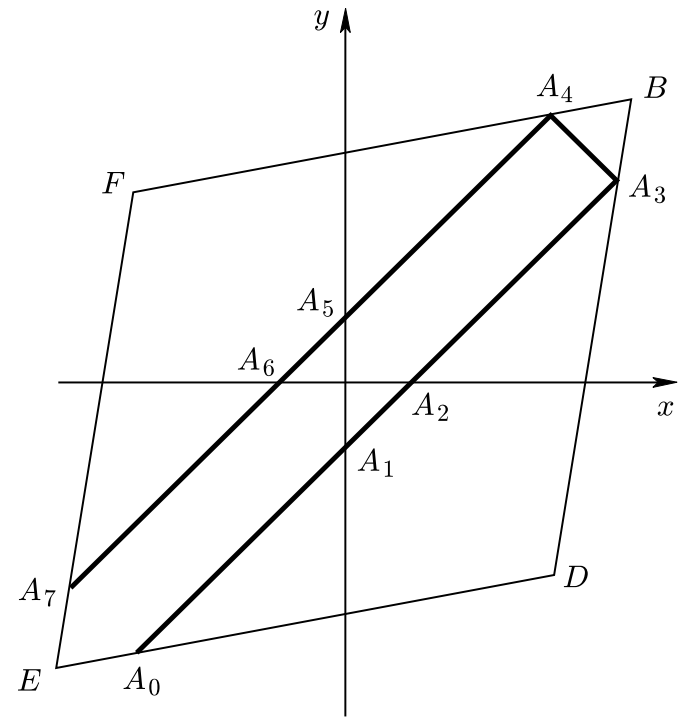

Рис. 1

Обозначим через $s(A)$ расстояние вдоль $L_{\alpha}$ от $A_{0}$ до $A \in L_{\alpha}$, и пусть $s_{j}=s\left(A_{j}\right)$, $s_{\Phi}(j)=s\left(\Phi\left(A_{j}\right)\right), j=\overline{0,7}$. Очевидно, $s_{0}=0, s_{2}-s_{1}=s_{4}-s_{3}=s_{6}-s_{5}=\alpha \sqrt{2}$. Так каK

$A_{0}=\left(-1+(a+1) \frac{\alpha}{2},-1+(a-1) \frac{\alpha}{2}\right), \quad \Phi\left(A_{0}\right)=\left(1-a+\left(a^{2}+1\right) \frac{\alpha}{2}, 1-a-\alpha+\left(a^{2}+1\right) \frac{\alpha}{2}\right)$, то $s_{\Phi}(0)=(2-a+a(a-1) \alpha / 2) \sqrt{2}$ и, значит, $s_{\Phi}(0)>0$ при $0<\alpha<1 / 2$ и $1<a<2$. Учитьвая, что $s_{2}=[1-(a-1) \alpha / 2] \sqrt{2}$ и $2-a+a(a-1) \alpha / 2<1-(a-1) \alpha / 2$ при $0<\alpha<1 / 2$ и $1<a<2$, видим, что при данных $a$ и $\alpha \Phi\left(A_{0}\right) \in \operatorname{Int}\left(A_{0} A_{2}\right)$. В силу симметрии то же самое справедливо для $\Phi\left(A_{7}\right)$ (т.е. $\Phi\left(A_{7}\right) \in \operatorname{Int}\left(A_{5} A_{7}\right)$ ). Следовательно, $\Phi\left(L_{\alpha}^{+}\right) \subset L_{\alpha}$ и $\Phi\left(L_{\alpha}^{-}\right) \subset L_{\alpha}$ при таких $a$ и $\alpha$. Вследствие этого $\Phi$ порождает разрьвное кусочно-линейное монотонное отображение

$$
\varphi: L_{\alpha}^{+} \cup L_{\alpha}^{-} \rightarrow L_{\alpha}
$$

такое, что

$$
\begin{aligned}
& \varphi\left(A_{0} A_{1}\right)=\varphi\left(A_{4} A_{5}\right)=\varphi\left(A_{0}\right) A_{3} \subset L_{\alpha}^{-}, \\
& \varphi\left(A_{2} A_{3}\right)=\varphi\left(A_{6} A_{7}\right)=A_{4} \varphi\left(A_{7}\right) \subset L_{\alpha}^{+}, \\
& \varphi\left(A_{1} A_{2}\right)=\varphi\left(A_{5} A_{6}\right)=A_{3} A_{4}=L_{\alpha}^{\star} .
\end{aligned}
$$

Обозначим $S=\varphi(s)$ функцию, задающую отображение $\varphi$. Ее график изображен на рис. 2 и состоит из двух параллельных ломаных, каждая из которьх включает в себя три отрезка прямых, из которых крайние наклонены под одинаковым углом к оси $s$, а средний параллелен диагонали $S=s$. Ломаные разделены интервалом (длины $\alpha \sqrt{2}$ ), на котором отображение $\varphi$ не определено. Обозначим через $\gamma$ тангенс угла наклона 


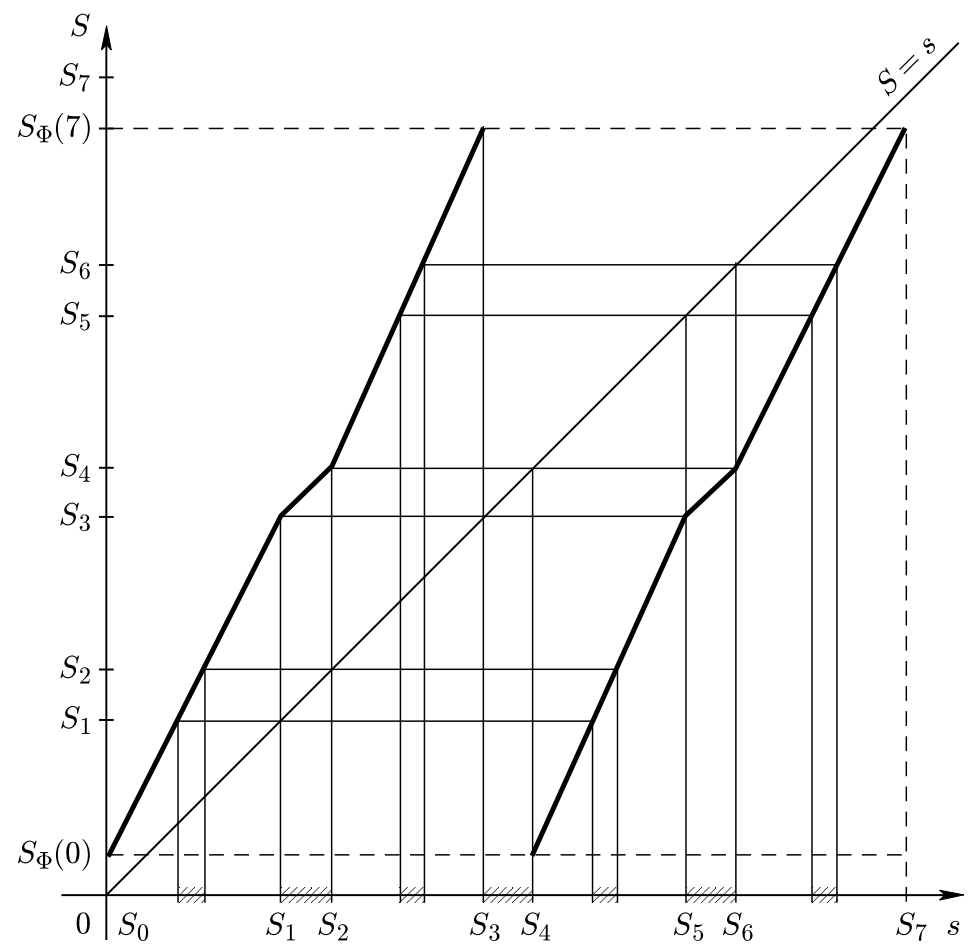

Рис. 2

крайних сегментов ломаных к оси $s$. Очевидно, что $\gamma=\left(s_{\Phi}(1)-s_{\Phi}(0)\right) / s_{1}$. Поскольку $s_{\Phi}(1)-s_{\Phi}(0)=s_{3}-s_{\Phi}(0)=2 s_{1}+\alpha \sqrt{2}-s_{\Phi}(0)$, то $\gamma=2+\left(\alpha \sqrt{2}-s_{\Phi}(0)\right) / s_{1}$. Учитывая, что $s_{1}=[1-(a+1) \alpha / 2] \sqrt{2}$ и что $s_{\Phi}(0)=(2-a+a(a-1) \alpha / 2) \sqrt{2}$, отсюда находим, что $\gamma=a(<2$ для $1<a<2)$.

Tеорема 2. Если $a \in(1,2) u \zeta=(a-1) /(2 a)$, mо существуют $\alpha \in(0,1 / 2)$ maкие, что на $L_{\alpha} \backslash L_{\alpha}^{\star}$ найдется нигде не плотное, непустое, замкнутое, инвариантное относительно $\Phi$ мнохество $C_{a \alpha} \neq T_{+}^{(\alpha)} \cup T_{-}^{(\alpha)}$. Более того, если $a>\sqrt{2}$, то среди наборов а и а имеются такие пары значений, что соответствующие им $C_{a \alpha}$ - несчетные мноэсества, хаотичные в смысле Ли и Йорка [8], а действие $\Phi$ топологически перемешивает каждое из них.

ДокАЗАТЕЛЬСТво. Приступим к построению $C_{a \alpha}$. Поскольку

$$
\Phi\left(\Phi\left(A_{0}\right) A_{3}\right)=\Phi^{2}\left(A_{0}\right) \Phi\left(A_{7}\right) \quad \text { и } \Phi\left(A_{4} \Phi\left(A_{7}\right)\right)=\Phi\left(A_{0}\right) \Phi^{2}\left(A_{7}\right),
$$

то $\Phi\left(\Phi\left(A_{0}\right) A_{3} \cup A_{4} \Phi\left(A_{7}\right)\right)=\Phi\left(A_{0}\right) \Phi\left(A_{7}\right) \stackrel{\text { def }}{=} \widehat{L}_{\alpha} \subset L_{\alpha}$ и, значит,

$$
\Phi\left(\widehat{L}_{\alpha} \backslash \operatorname{Int}\left(A_{3} A_{4}\right)\right)=\widehat{L}_{\alpha}
$$

Обозначим через $\widehat{\Phi}$ ограничение $\Phi$ на $\widehat{L}_{\alpha} \backslash L_{\alpha}^{\star}$ и рассмотрим

$$
C_{a \alpha}=\widehat{L}_{\alpha} \backslash \bigcup_{j=0}^{\infty} \widehat{\Phi}^{-j}\left(\operatorname{Int}\left(A_{3} A_{4}\right)\right) .
$$


Очевидно, что $C_{a \alpha}$ - замкнутое инвариантное относительно $\Phi$ множество. Очевидно, $T_{ \pm}^{(\alpha)} \in C_{a \alpha}$. Покажем, что $C_{a \alpha} \neq T_{-}^{(\alpha)} \cup T_{+}^{(\alpha)}$. Заметим, прежде всего, что поскольку $\widehat{\Phi} \equiv \varphi$, то имеется взаимно-однозначное соответствие между $C_{a \alpha}$ и множеством, которое образуется на отрезке $\left[s_{\Phi}(0), s_{\Phi}(7)\right]$ в процессе последовательного удаления прообразов интервала $\left(s_{3}, s_{4}\right)$. Последнее для простоты мы будем назьвать тоже $C_{a \alpha}$. Этот процесс (на рис. 2 изображены его первые три этапа) по форме напоминает процесс построения множества Кантора. При этом левая ломаная определяет положение прообразов $\left(s_{3}, s_{4}\right)$, удаляемых из $\left[s_{\Phi}(0), s_{3}\right]$, а правая ломаная - положение прообразов $\left(s_{3}, s_{4}\right)$, удаляемых из $\left[s_{4}, s_{\Phi}(7)\right]$. Очевидно, что прообразы $j$-го и $(j+k)$-го порядка отрезка $\left(s_{3}, s_{4}\right)$, где $k$ - произвольное натуральное число, не пересекаются. (В самом деле, допустим противное, т.е. существует интервал $\varnothing \neq \Delta=\left(\bar{s}_{j}, \overline{\bar{s}}_{j}\right) \cap\left(\bar{s}_{j+k}, \overline{\bar{s}}_{j+k}\right)$, где $\left(\bar{s}_{m}, \overline{\bar{s}}_{m}\right) \in \varphi^{-m}\left(\left(s_{3}, s_{4}\right)\right), m=j, j+k$. Тогда, с одной стороны, $\varphi^{j}(\Delta) \subset\left(s_{3}, s_{4}\right)$, a с другой, $\varphi^{j}(\Delta) \subset \varphi^{-k}\left(\left(s_{3}, s_{4}\right)\right) \subset\left[s_{\Phi}(0), s_{3}\right] \cup\left[s_{4}, s_{\Phi}(7)\right]$, что невозможно.)

Покажем, что при любом $1<a<2$ выполнено $C_{a \alpha} \neq T_{-}^{(\alpha)} \cup T_{+}^{(\alpha)}$ по крайней мере для некоторых $0<\alpha<1 / 2$.

ЛЕмма 1. Для каждого $a \in(1,2)$ существует как минимум два натуральных $N>1$ таких, что для каждого из них найдется $\alpha_{a, N} \in(0,1 / 2)$ такое, что отображсение $\Phi$ на $L_{\alpha_{a, N}}$ имеет иикл периода $2 N$.

ДоКАЗАТЕЛЬСтВо. В силу симметрии ясно, что если $\Phi^{n+1}\left(A_{0}\right)=A_{1}$, то $\Phi^{n+1}\left(A_{7}\right)$ $=A_{6}$, и наоборот. Будем искать цикл вида

$$
\begin{aligned}
\Phi\left(A_{0}\right) \stackrel{\Phi^{n}}{\longrightarrow} \Phi^{n+1}\left(A_{0}\right) & =A_{1} \stackrel{\Phi}{\longrightarrow} A_{3} \stackrel{\Phi}{\longrightarrow} \Phi\left(A_{7}\right) \stackrel{\Phi^{n}}{\longrightarrow} \Phi^{n+1}\left(A_{7}\right) \\
& =\Phi\left(A_{6}\right) \stackrel{\Phi}{\longrightarrow} A_{4} \stackrel{\Phi}{\longrightarrow} \Phi\left(A_{0}\right),
\end{aligned}
$$

где $n=N-2$. Так как $\gamma=a$ и $s_{\Phi}(0)=(2-a+a(a-1) \alpha / 2) \sqrt{2}$, то

$$
s_{\Phi^{n+1}}(0)=a^{n}(2-a+a(a-1) \alpha / 2) \sqrt{2} .
$$

Тогда из условия $\Phi^{n+1}\left(A_{0}\right)=A_{1}$ получаем следующее уравнение для определения значения $\alpha_{a, n+2}$ :

$$
a^{n}\left[2-a+\frac{a(a-1)}{2} \alpha_{a, n+2}\right] \sqrt{2}=\left[1-\frac{a+1}{2} \alpha_{a, n+2}\right] \sqrt{2} .
$$

Решая его, находим, что

$$
\alpha_{a, n+2}=\frac{2\left[1-a^{n}(2-a)\right]}{a^{n+1}(a-1)+a+1} .
$$

Подставляя сюда $n=0$ и 1 , имеем

$$
\alpha_{a, 2}=2 \frac{a-1}{a^{2}+1}, \quad \alpha_{a, 3}=\frac{(a-1)^{2}}{a^{2}(a-1)+(a+1)} .
$$

Нетрудно убедиться, что с ростом $а$ значения $\alpha_{a, 2}, \alpha_{a, 3}$ растут и $0<\alpha_{a, 2}<2 / 5$, $0<\alpha_{a, 3}<2 / 7$, когда $1<a<2$.

Заметим, что, поскольку с ростом $a$ и $n$ значения выражения $a^{n+1}(a-1)+a+1$ растут, a $\lim _{a \rightarrow 2-}\left[1-a^{n}(2-a)\right]=0$ для любого (фиксированного) $n$, то при $a \rightarrow 2-$ количество пар $N, \alpha_{a, N}$, удовлетворяюших условиям леммы, неограниченно растет. 
Лемма 2. Множество $C_{а \alpha}$ нигде не плотно.

ДокаЗАтЕЛьСтво. Допустим противное, т.е. что существует $[\bar{s}, \overline{\bar{s}}] \subset C_{a \alpha}, s_{\Phi}(0)<$ $\bar{s}<\overline{\bar{s}}<s_{\Phi}(7)$. Поскольку $\varphi: C_{a \alpha} \rightarrow C_{a \alpha}$, то для любого $j \varphi^{j}([\bar{s}, \overline{\bar{s}}]) \subset C_{a \alpha}$. А так как $\varphi$ растягивает произвольный отрезок в $\gamma>1$ раз, то длина $\varphi^{j}([\bar{s}, \overline{\bar{s}}])$, равная $\gamma^{j}|\overline{\bar{s}}-\bar{s}| \rightarrow \infty$ при $j \rightarrow \infty$. Но это значит, что найдется $J$ такое, что $\varphi^{J}([\bar{s}, \overline{\bar{s}}])=\left[s_{\Phi}(0), s_{\Phi}(7)\right]$. Последнее невозможно, поскольку по построению $C_{a \alpha} \neq\left[s_{\Phi}(0), s_{\Phi}(7)\right]$.

ЛЕмма 3. Если а и а таковы, что $\Phi^{J+1}\left(A_{0}\right)=T_{-}^{(\alpha)}\left(\Phi^{J+1}\left(A_{7}\right)=T_{+}^{(\alpha)}\right)$, mо $\Phi-$ отобрахение, топологически перемешивающее $C_{a \alpha}$.

ДокАЗАТЕЛЬСТво. Пусть $\widehat{s} \in C_{a \alpha}, \xi>0$-произвольно малая константа и $\widehat{U}_{\xi}(s) \stackrel{\text { def }}{=}$ $U_{\xi}(\widehat{s}) \cap C_{a \alpha}-\xi$-окрестность $\widehat{s}$ в $C_{a \alpha}$. Ввиду нигде не плотности $C_{a \alpha}$ из алгоритма построения $C_{a \alpha}$ вытекает, что для данного $\xi$ найдется натуральное $M=M(\xi)$ такое, что $\varphi^{-M}\left(s_{4}\right) \cap \widehat{U}_{\xi}(s) \neq \varnothing$. Поскольку $\varphi\left(s_{4}\right)=s_{\Phi}(0)$ и $\varphi^{J}\left(s_{\Phi}(0)\right)=s\left(T_{-}^{(\alpha)}\right)$, это значит, что существует $\eta>0$ такое, что

$$
\widehat{U}_{\eta}\left(s\left(T_{-}^{(\alpha)}\right)\right) \subset \varphi^{M+J+1}\left(U_{\xi}(\widehat{s})\right)
$$

A так как

$$
\varphi^{2}\left(C_{a \alpha} \cap\left(\left[0, s_{1}\right] \cup\left[s_{2}, s_{3}\right]\right)\right)=C_{a \alpha} \cap\left(\left[0, s_{1}\right] \cup\left[s_{2}, s_{3}\right]\right)
$$

и $\varphi$ - растягивающее на $C_{a \alpha}$ отображение, то найдется натуральное $I=I(\eta)$ такое, что $\varphi^{2 I}\left(\widehat{U}_{\eta}\left(s\left(T_{-}^{(\alpha)}\right)\right)\right)=C_{a \alpha} \cap\left[0, s_{3}\right]$. Отсюда вытекает, что $\varphi^{M+J+2 I+2}\left(\widehat{U}_{\xi}(\widehat{s})\right) \supset C_{a \alpha}$. Последнее означает, что $\varphi$ топологически перемешивает $C_{a \alpha}$.

Лемма 4. Для каждого $\sqrt{2}<a<2$ найдутся иелое $n \geqslant 0 u \alpha_{a, n+2} \in(0,1 / 2)$ такие, что

$$
\Phi^{n+2}\left(A_{0}\right)=T_{-}^{(\alpha)}, \quad \Phi^{n+3}\left(A_{0}\right)=T_{+}^{(\alpha)} .
$$

ДокАЗАТЕЛЬСТВо. В процессе доказательства леммы 1 установлено, что для каждого $1<a<2$ и $n=0$ или 1 найдется $\alpha_{a, n+2} \in(0,1 / 2)$ такое, что $\Phi^{n+1}\left(A_{0}\right)=A_{1}$, $\Phi^{n+2}\left(A_{0}\right)=A_{3}$. Пусть $n=0$. Обозначим

$$
t=\left(\frac{\alpha}{2}-\frac{1}{1+a},-\frac{\alpha}{2}-\frac{1}{1+a}\right)
$$

- прообраз точки $T_{-}^{(\alpha)}$ на $L_{\alpha}^{-}$, а $s_{t}=(1 /(1+a)-\alpha / 2) a \sqrt{2}-$ расстояние от $A_{0}$ до $t$. Заметим теперь, что так как в лемме 1 доказано существование $\alpha_{a, 2}$ такого, что $\Phi\left(A_{0}\right)=A_{1}$, то в таком случае (т.е. при этом значении $\alpha) s_{\Phi}(0)>s_{t}$. Ипоскольку $s_{\Phi}(0), s_{t}-$ функции непрерывные по $\alpha$, лемма будет доказана, если мы сумеем показать, что $s_{\Phi}(0)<s_{t}$ при $\alpha=0$. Нетрудно проверить, что это требование эквивалентно выполнению неравенства $a /(1+a)>2-a+\alpha a^{2} / 2$ при $\alpha=0$. Последнее справедливо, когда $a>\sqrt{2}$.

Лемма 5. Для каждого $\sqrt{2}<a<2$ существует по крайней мере одно $0<\alpha<1 / 2$ такое, что пара точек $T_{-}^{(\alpha)}, T_{+}^{(\alpha)}-$ репеллер с мгновенным возвра$\operatorname{moм}[9]$. 
ДокАЗАТЕЛЬСтво. В предыдущей лемме мы показали существование для каждого $a \in(\sqrt{2}, 2)$ такого $\alpha \in(0,1 / 2)$, что $\Phi^{2}\left(A_{0}\right)=T_{-}^{(\alpha)}, \Phi^{3}\left(A_{0}\right)=T_{+}^{(\alpha)}$. А так как коэффициент наклона графика отображения, обратного к ограничению $\Phi$ на $L_{\alpha}$ (которое, как не взаимно-однозначное, имеет, естественно, несколько ветвей), равен $1 / a<1$, то существует последовательность прообразов точки $A_{0}$, которые сходятся к $T_{-}^{(\alpha)} \cup T_{+}^{(\alpha)}$. С учетом гиперболичности точек $T_{-}^{(\alpha)}, T_{+}^{(\alpha)}$ это значит, что они являются репеллером с мгновенным возвратом.

Вернемся к доказательству теоремы 2. Первая часть утверждения теоремы- очевидное следствие лемм 1 и 2 . Что же касается второй части, то с учетом результата Маротто (см., например, [9]-[12]) ее справедливость является прямым следствием лемм 3-5.

Из вышесказанного вытекает еше такое

СлЕДСТВИЕ. Если $a \in(1,2) u \zeta=(a-1) /(2 a)$, то $\Lambda$-множество, устойчивое по Ляпунову.

\section{СПИСОК ЦИТИРОВАННОЙ ЛИТЕРАТУРЫ}

[1] Pikovsky A.S., Grassberger P. Symmetry breaking bifurcation for coupled chaotic attractors // J. Phys. A. Math. Gen. 1991. V. 24. P. 4587-4597.

[2] Pikovsky A.S., Kurths J. Collective behavior in ensembles of globally coupled maps // Physica D. 1994. V. 76. P. 411-419.

[3] Losson J., Mackey M. Coupling induced statistical cycling in two diffusively coupled maps // Physica D. 1994. V. 72. P. 324-342.

[4] Keller G. Mixing for Finite Systems of Coupled Tent Maps // Preprint ESI 388. Vienna, 1996.

[5] Добрынский В.А. Существование двумерных топологически перемешивающих аттракторов у некоторых кусочно-линейных отображений плоскости // Изв. РАН. Сер. матем. 1998. T. 62 . №6. C. $53-58$.

[6] Добрынский В.А. Критические множества и унимодальные отображения квадрата // Докл. РАН. 1995. Т. 341. № 4. С. 442-445.

[7] Добрынский В.А. Критические множества и унимодальные отображения квадрата // Матем. заметки. 1995. Т. 58. № 5. С. 669-680.

[8] Li T.-Y., Yorke J. A. Period three implies chaos // Amer. Math. Monthly. 1975. V. 82. P. 985-992.

[9] Marotto F. R. Snap-back repellers imply chaos in $\mathbb{R}^{n}$ // J. Math. Anal. Appl. 1978. V. 63. P. 199-223.

[10] Rogers T.D., Marotto F. R. Perturbations of mappings with periodic repellers // Nonlinear Analysis. 1983. V. 7. № 1. P. 97-100.

[11] Hale J. K., Lin X. B. Symbolic dynamics and nonlinear semiflows // Ann. Mat. Pura Appl. 1986. V. 144. P. 229-259.

[12] Steinlein H., Walters H. O. Hyperbolic sets, transversal homoclinic trajectories and symbolic dynamics for $C^{1}$-maps in Banach spaces // J. Dynamics and Differential Equations. 1990. V. 2. № 3. P. 325-365.

Институт математики НАН Украины, г. Киев

E-mail: dobry@imath.kiev.ua

Поступило 20.01.2001 